\title{
Location and the functionality of erythropoietin receptor(s) in $\mathbf{A} 2780$ cells
}

\author{
PETER SOLÁR ${ }^{1}$, GABRIELA HRČKOVÁ ${ }^{4}$, LENKA VARINSKÁ ${ }^{2}$, ZUZANA SOLÁROVÁ $^{3}$, JÁN KRIŠKA ${ }^{1}$, \\ IVANA UHRÍNOVÁ ${ }^{1}$, MARTIN KELLO ${ }^{1}$, JÁN MOJŽIŠ ${ }^{2}$, PETER FEDOROČKO ${ }^{1}$ and ARTHUR J. SYTKOWSKI ${ }^{5,6}$ \\ ${ }^{1}$ Laboratory for Cell Biology, Institute of Biology and Ecology, Faculty of Science, ${ }^{2}$ Department of Pharmacology, and \\ ${ }^{3}$ Geriatric Nursing Clinic, Faculty of Medicine, P.J. Šafárik University; ${ }^{4}$ Institute of Parasitology, Slovak Academy of \\ Sciences, 04001 Košice, Slovak Republic; ${ }^{5}$ Laboratory for Cell and Molecular Biology, Beth Israel Deaconess \\ Medical Center, Department of Medicine, Harvard Medical School, Boston, MA 02215; ${ }^{6}$ Hematology and \\ Oncology Group, Medical and Scientific Services, Quintiles Transnational, Arlington, MA 02476, USA
}

Received March 2, 2012; Accepted April 2, 2012

DOI: 10.3892/or.2012.1795

\begin{abstract}
Erythropoietin (Epo) is a critical regulator of erythroid cell proliferation, differentiation and apoptosis. In the form of a recombinant protein, it is widely used to treat various forms of anemia, including that associated with cancer and with the myelosuppressive effects of chemotherapy. Studies of ovarian cancer cell lines have demonstrated the presence of the Epo receptor (EpoR), but there are disagreements regarding its localization and functionality in these cells. Using fluorescence microscopy, we were not able to identify the EpoR on the surface of A2780 cells, in contrast to the positive control K562 cells. Flow cytometry did reveal a weak surface EpoR signal in A2780 cells. Interestingly, most of the EpoR in A2780 cells was found in the cytoplasm, more abundantly as an intracellular membraneassociated protein than a soluble one. Silencing EpoR expression by lentiviral-mediated shRNA resulted in reduced A2780 proliferation as well as reduction in Epo-induced phosphorylation of Erk1/2. Our findings provide important insights into the biology of the EpoR in ovarian cancer cells.
\end{abstract}

\section{Introduction}

Erythropoietin (Epo) is a glycoprotein hormone essential for erythroid progenitor cell survival, proliferation and differentiation. Expression of the Epo gene is regulated by tissue oxygen concentration/hypoxia and, thus, Epo is important throughout the body by virtue of its control of red blood cell production.

Correspondence to: Dr Arthur J. Sytkowski, Laboratory for Cell and Molecular Biology, Beth Israel Deaconess Medical Center, Department of Medicine, Harvard Medical School, 330 Brookline Ave., FA-824, Boston, MA 02215, USA

E-mail: asytkows@bidmc.harvard.edu

Key words: erythropoietin receptor, ovarian cancer, A2780, proliferation, Erk1/2
Recombinant Epo (rhEpo) has been used clinically for over two decades for the treatment of various forms of anemia, including anemia associated with malignancies.

Epo stimulates cells through its interaction with the Epo receptor (EpoR) on the cell surface. The EpoR has been identified not only in hematopoietic cells, but also in many non-hematopoietic cells and tissues where autocrine, paracrine and endocrine actions have been proposed (1). The presence of EpoR in cancer cells and tissues has raised important questions regarding the potential consequences of recombinant Epo use in cancer patients $(2,3)$.

According to McKinney and Arcasoy (4), there are two potential mechanisms by which rhEpo therapy may promote tumor progression and reduce survival in some cancer patients: i) rhEpo therapy may exert local effects in tumors, acting directly on tumor cells or other cell types in the tumor microenvironment, such as the vascular endothelium and tumor-associated macrophages; or ii) rhEpo may cause effects that indirectly alter tumor biology in an unfavorable manner or directly give rise to specific systemic toxicities that impair survival. In this regard, elevated hemoglobin, increased viscosity, platelet activation, endothelial progenitor mobilization, immunomodulatory effects and others could play significant roles.

Different types of tumors as well as cell lines have been found to express EpoR mRNA transcripts, which might be translated into full-length EpoR as well as soluble or other truncated forms (5). In this regard, Um et al (6) measured internalized ${ }^{125} \mathrm{I}-\mathrm{Epo}$ and found that just 50 high affinity cell surface Epo binding sites were sufficient for Epo-mediated activation of intracellular signal transduction in SH-SY5Y and PC-12 cancer cells. EpoR expression has been demonstrated by flow cytometry using a specific EpoR antibody in a panel of 29 tumor cell lines, including 18 adherent cell lines (7). Other investigators, using conventional measurements of radiolabeled ${ }^{125} \mathrm{I}-\mathrm{Epo}$, could not detect cell surface EpoR in adherent tumor cell lines in spite of detection by Western immunoblotting of more than 400 dimers of EpoR/cell (8).

Several groups have reported the presence of EpoR in ovarian cancer cell lines (7-10), but there are disagreements, especially 
regarding the disproportion in mRNA concentration and the amount of surface EpoR protein and/or EpoR localization. Also, the functionality of these EpoR has been in dispute. Therefore, we have carried out a series of experiments to fully demonstrate the cellular localization and functionality of the EpoR in A2780 ovarian cancer cells.

\section{Materials and methods}

Cell lines. Human ovarian adenocarcinoma cell line A2780 and human erythromyeloblastoid leukemia cell line K562 were obtained from the American Tissue Culture Collection. Both cell lines were grown in RPMI-1640 with L-glutamine (Invitrogen Co., Carlsbad, CA, USA). Medium was supplemented with $10 \%$ FCS and antibiotic/antimycotic solution $(100 \mathrm{U} / \mathrm{ml}$ penicillin, $100 \mu \mathrm{g} / \mathrm{ml}$ streptomycin, and $0.25 \mu \mathrm{g} / \mathrm{ml}$ amphotericin $\mathrm{B}$; Invitrogen Co.). The cells were maintained under standard tissue culture conditions of $37^{\circ} \mathrm{C}, 95 \%$ air $/ 5 \% \mathrm{CO}_{2}$. The number of cells was determined using a Coulter counter (Model ZF, Coulter Electronics Ltd., Luton, Beds., UK) and total cell viability was analyzed by staining with $0.15 \%$ eosin followed by light microscopy.

FACSCalibur analysis of EpoR. A2780 and K562 cells were harvested and Fc-blocked by treatment with $1 \mu \mathrm{g}$ of human $\mathrm{IgG} / 10^{5}$ cells for $15 \mathrm{~min}$ at room temperature. The cells were then incubated with $10 \mu \mathrm{l}$ of fluorescein-conjugated anti-EpoR antibody (FAB307F, R\&D Systems, Minneapolis, MN, USA) for $45 \mathrm{~min}$ at $4^{\circ} \mathrm{C}$, followed by washing of cells twice in PBS. For isotype negative control staining we used mouse IgG2B control antibody (IC0041F, R\&D Systems). Finally, cells were resuspended in $300 \mu 1$ of PBS and analyzed using a FACSCalibur flow cytometer (Becton-Dickinson, San Diego, CA, USA). EpoR presence on A2780 and K562 cells was expressed as the ratio of the fluorescence median intensity in non-trypsinized cells compared to trypsinized controls.

EpoR silencing. For EpoR knockdown, A2780 cells were transduced by pseudoviral particles containing the lentiviral short hairpin RNA (shRNA) targeting the sequence CGGTGC GCTTCTGGTGTTC of EpoR mRNA. shRNA targeting EpoR as well as non-targeting shRNA vector (NTshRNA) were packaged by 293Ta cells through their transfection using Lenti-Pac FIV mix and EndoFectin Lenti (GeneCopoeia, Rockville, MD, USA). The ratio of $3.0 \mu \mathrm{l}$ of EndoFectin Lenti per $1 \mu \mathrm{g}$ of plasmid has been found to be optimal for transfection. Pseudovirus containing culture medium was collected $48 \mathrm{~h}$ post-transfection of 293Ta cells and after centrifugation and filtration $(0.45 \mu \mathrm{m}$ PES low protein-binding filters) was immediately titered, aliquoted and stored at $-80^{\circ} \mathrm{C}$.

MTT assay. Control A2780 cells as well as shRNA and NTshRNA transduced A2780 cells were seeded into 96-well cell culture plates at a density of 5,000 cells/well. Later, at 24, 48, 72 and 96 h MTT (3-(4,5-dimethyl thiazol-2-yl)-2,5-diphenyl tetrazolium bromide; Sigma Chemical Co., St. Louis, MO, USA) was added $(0.2 \mathrm{mg} / \mathrm{ml})$, followed by $4 \mathrm{~h}$ cell incubation under standard culture conditions. Thereafter, violet-blue crystals of metabolized products were dissolved using sodium dodecyl sulfate (SDS; 3.3\%) (Sigma Chemical Co.). The absorbance measurements were carried out using a FLUOStar Optima universal microplate reader (BMG Labtechnologies, Offenburg, Germany). The blank was subtracted, and results were expressed as a percentage of untreated control and presented as mean \pm SD of three independent experiments.

Immunofluorescence. K562 and A2780 cells were removed from tissue flasks by cell scraper and transferred into $50 \mathrm{ml}$ tubes. Both cell lines were processed using three protocols. In the first protocol, cell suspensions were washed with PBS containing $0.5 \%$ albumin and $0.1 \%$ sodium azide according to the protocol described by Johnstone and Thorpe (11). Cells were not fixed and incubation with primary anti-human EpoR antibody (1:100, AF-322-PB, R\&D Systems) was performed in the same buffer for $1 \mathrm{~h}$ at room temperature. After washing cell suspensions with PBS, secondary anti-goat IgG-FITC antibody (F0109, R\&D Systems) diluted in the same PBS (1:100) was added and cells were incubated for $1 \mathrm{~h}$ at RT. Cells pellets, after being washed extensively with PBS (containing $0.5 \%$ albumin, $0.1 \%$ Triton X-100, Sigma Chemical Co.) were dispersed in antifade mounting fluid containing 1,4-diazabicyclo $(2,2,2)$ octane (DABCO, Sigma Chemical Co.) and glycerol in PBS, placed on glass slides SuperFrost Plus (Menzel Gläser, Braunschweig, Germany) and immunofluorescence was detected using a Leica DMI 6000 fluorescent microscope at magnification x600.

The second protocol of cell processing allowed visualisation of integral antigens. K562 and A2780 cell pellets were resuspended in $4 \%$ cold paraformaldehyde in PBS without sodium azide and fixed at $4^{\circ} \mathrm{C}$ for $10 \mathrm{~min}$. After washing twice for 5 min in PBS containing $0.05 \%$ saponin and $0.5 \%$ BSA (Sigma Chemical Co.), cells were incubated with primary antibody followed by secondary antibody as described above. Finally, the presence of EpoR on the endoplasmic reticulum (ER) for potential localisation was investigated in both cell lines using double fluorescent staining. Briefly, both cell lines were transduced by CellLight reagents (Invitrogen Co.) and in the successfully transformed cells, ER was visualised by the expression of ER specific GFP labelled protein. EpoR staining was performed in transduced cells after the fixation of cells in 4\% cold paraformaldehyde. Incubation with biotinylated anti-human EpoR antibody (1:50, BAF307, R\&D Systems) was carried out overnight at $4^{\circ} \mathrm{C}$ followed by the incubation with streptavidine AlexaFluor 594 conjugate (1:50, S32356, Invitrogen Co.) for $2 \mathrm{~h}$ at $4^{\circ} \mathrm{C}$. We used fluorescent microscope Leica DMI 6000 at magnification x600 for visualisation and co-localisation of ER and EpoR in K562 and A2780 cells and the confocal laser scanning microscope Leica TCS ST5X for more detailed view of such a co-localisation in A2780 cells.

Extraction of membrane and cytoplasm protein fractions. To separate water-soluble proteins in cytoplasm from lipid-soluble proteins in cell membranes, protein extraction was performed according to the modified method described by Griffiths et al (12). Briefly, K562 and A2780 were harvested into $0.5 \mathrm{ml}$ of the extraction solution (10 mM Tris- $\mathrm{HCl}, \mathrm{pH} 8.0,2 \mathrm{mM} \mathrm{MgCl}_{2}$, $10 \mathrm{mM} \mathrm{KCl}, 1 \mathrm{mM}$ DTT, $0.1 \%$ SDS) with $50 \mu \mathrm{l}$ of diluted protease inhibitor cocktail (Sigma Chemical Co.). Cells were disrupted by sonication (Bandelin Sonopuls HD 2070) for $5 \mathrm{~min}$ at $20 \mathrm{kHz}$ and tubes with lysed cells were incubated on ice for $1 \mathrm{~h}$ under gentle shaking. Samples were centrifuged at $14,000 \mathrm{~g}, 4^{\circ} \mathrm{C}$ 
for $25 \mathrm{~min}$. Supernatants were collected and frozen at $-20^{\circ} \mathrm{C}$ and the remaining pellets were resuspended in $0.3 \mathrm{ml}$ of the lipidsoluble extraction solution of the same composition, except the SDS concentration (2\%) allowing solubilisation of the membrane proteins. Pellets were intensively shaken on ice for $2 \mathrm{~h}$ and centrifuged as before.

Extraction of cytoplasm and membrane proteins from K562 and A2780 cells was also performed using ProteoJET membrane protein extraction kit (no. K0321, ThermoFisher Scientific, St. Leon-Rot, Germany). Briefly, $5 \times 10^{6}$ cells were permeabilized with $1.5 \mathrm{ml}$ of ice-cold cell permeabilization buffer to release the cytoplasm protein fraction. The cell debris was then treated with $1 \mathrm{ml}$ of ice-cold membrane protein extraction buffer which selectively solubilized the majority of integral and membraneassociated proteins and isolated them after a 30-min incubation at $4^{\circ} \mathrm{C}$ as a separate fraction. Protein fractions isolated by both protocols were quantified by Lowry assay and analysed by Western blot analysis.

Western blot analyses. Western blot analyses were carried out according to the standard protocol. A2780 cells transduced by shRNA or NTshRNA were serum starved overnight and incubated with Epo for $30 \mathrm{~min}$. Then the cells were washed twice with ice-cold PBS and scraped into RIPA buffer (PBS, 1\% Nonidet P-40, $0.5 \%$ sodium deoxycholate, $0.1 \%$ SDS; all Sigma Chemical Co.) containing freshly added protease and phosphatase inhibitor cocktail (Roche Diagnostics, Penzberg, Germany). Scraped lysates were transferred into a microcentrifuge tube and passed through a 21-gauge needle to shear the DNA. After incubation of the lysates on ice for $45 \mathrm{~min}$, the samples were centrifuged at $10,000 \mathrm{~g}$ for $10 \mathrm{~min}$ at $4^{\circ} \mathrm{C}$ and the supernatant was transferred into a new microcentrifuge tube. The protein samples were separated on $10 \%$ SDS-PAGE gels, electroblotted onto Immobilon-P transfer membrane (Millipore Co., Billerica, MA, USA) and incubated using the following primary antibodies: anti-EpoR (AF-322-PB, 1:1,000, R\&D Systems), anti-p44/42 MAP kinase (no. 9102, 1:1,000, Cell Signaling Technology, Danvers, MA, USA), anti-phospho-p44/42 MAP kinase (no. 9272, 1:1,000, Cell Signaling Technology), anti-STAT5 (AF2168, 1:200, R\&D Systems), anti-phospho-STAT5 (Y694/Y699) (AF4190, 1:100, R\&D Systems), anti-Jak-2 (no. 3230, 1:1,000, Cell Signaling Technology), anti-phospho-Jak-2 (Tyr1007/1008) (no. 3776, 1:1,000, Cell Signaling Technology), anti-Epo (MAB2871, 1:500, R\&D Systems) and anti- $\beta$-actin (clone AC-74, 1:10,000, Sigma Chemical Co.). Then the membranes were incubated with secondary horseradish peroxidase-conjugated antibodies [goat anti-rabbit IgG F(ab9) 2, 1:10,000, PI-31461, goat anti-mouse IgG F(ab9) 2, 1:10,000, PI-31436 or rabbit anti-goat IgG F(ab9) 2, 1:10,000, PI-31403; Pierce, Rockford, IL, USA] for $1 \mathrm{~h}$, and the antibody reactivity was visualized with ECL Western blotting substrate (PI-32106, Pierce) using Kodak Biomax film (no. 1788207, Sigma Chemical Co.).

\section{Results}

Previous studies of EpoR and ovarian adenocarcinoma A2780 cells used cells donated by other users without necessarily showing the total number of passages made by assumed original (ATCC) cells. Thus, clonal differences in A2780 cells, including EpoR expression, might be expected. For the present study, we used A2780 cells descended from ATCC and used them for different analysis between the passages 23-25. Human erythromyeloblastoid leukemia cell line K562 served in EpoR localization studies as a positive control.

Fluorescence microscopy of EpoR in K562 cells showed plasma membrane localization (Fig. 1). This method did not demonstrate EpoR on A2780 cells. In contrast to K562, most of the EpoR in A2780 cells was found in the cytoplasm (Fig. 2A and B). Using flow cytometric analysis and comparing nontrypsinized cells to trypsinized controls, A2780 exhibited a weak but definite EpoR signal on the surface (Fig. 3). In fact, the amount of surface EpoR was lower in A2780 than in K562 cells, which seems to be contrary to the total amount of EpoR detected in whole cell lysates. Indeed, the total amount of EpoR detected by Western blot analysis was higher in A2780 cells than in K562 cells (data not shown).

Furthermore, the extraction of cytoplasm and membrane protein fractions by two independent techniques (detailed in Materials and methods) revealed a very interesting finding. K562 cells showed the expected EpoR presence in the membrane protein fraction. Surprisingly, at least in regard to the localization detected by fluorescent microscopy results, EpoR protein was also more widely detected in the membrane fraction of A2780 cells (Fig. 4). The verification of no cross contamination of protein fractions during extraction was done through detection of specific cytoplasm proteins $\beta$-actin and Erk1/2. Although $\beta$-actin was in minority present in membrane fraction, Erk1/2 protein was only detected in the cytoplasm of both cell lines (Fig. 4). Moreover, co-localization study with specific GFP labeled endoplasmic reticulum membrane protein and EpoR staining when using confocal microscopy showed EpoR presence on the membranes of endoplasmic reticulum but not exclusively on these membrane organelles (Fig. 2C and D). Therefore, there appear to be other membrane organelles or structures in A2780 cells where EpoR can be localized.

Taking all of our EpoR localization studies together, it appears that most of the EpoR protein in A2780 cells is present in the cytoplasm, not in a soluble form but rather as a membrane protein.

We investigated the functionality of the EpoR in A2780 cells through rhEpo-induced signal transduction of control as well as EpoR silenced cells. In the first analysis, control A2780 cells treated overnight with $50 \mathrm{IU} / \mathrm{ml}$ of rhEpo revealed strong signal in the form of phosphorylated Erk1/2 proteins. On the other hand, addition of soluble EpoR combined with rhEpo diminished this strong phosphorylation of Erk1/2. In this regard, no signal was detected after the administration of vehicle ( $0.1 \%$ of serum) only. Soluble EpoR induced weak phosphorylation of Erk1/2 proteins (Fig. 5). In the second analysis, we studied the effect of specific lentiviral mediated shRNA silencing of EpoR expression on Epo induced signal transduction of A2780 cells. EpoR down-regulation resulted in deprivation of Erk1/2 signalization induced by 30 min rhEpo treatment of serum starved NTshRNA control cells (Fig. 6). The same situation was observed after a 5-min treatment of cells with rhEpo (data not shown). Moreover, EpoR silencing reduced protein level of JAK2 as well as STAT5 proteins in comparison to NTshRNA control cells (Fig. 6). Although addition of rhEpo did not stimulate A2780 proliferation, down-regulating EpoR expression by shRNA did result in reduced proliferation. Indeed, A2780 cells with down-regulated 


\section{K 562 cells}
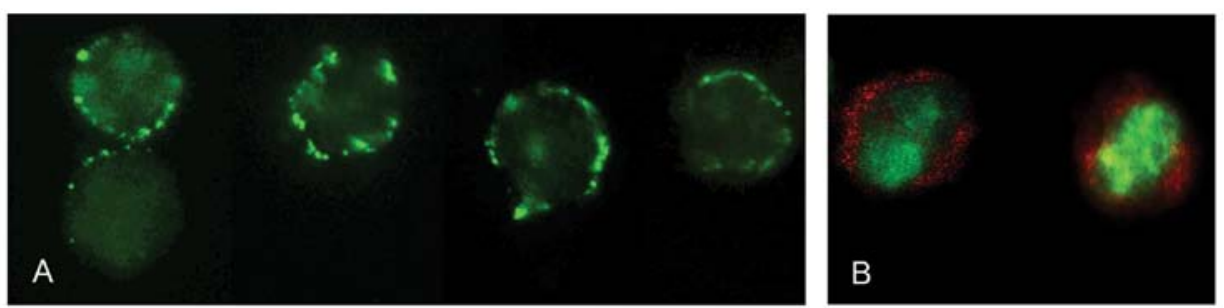

Figure 1. Immunocytochemical analysis of erythropoietin receptor and endoplasmic reticulum with secretory transport distribution in K562 cells. (A) Single fluorescent staining using antibody to erythropoietin receptor (EpoR) and FITC-labelled secondary antibody showing clear (discrete) distribution of receptor exclusively in the cytoplasmic membrane. (B) Double staining of EpoR with biotinylated anti-human EpoR antibody (followed by incubation with streptavidine AlexaFluor 594 conjugate) and endoplasmic reticulum (ER) and secretory transport specific GFP labelled protein expressed following transduction of cells with CellLight reagents (see Materials and methods). Representative picture with EpoR (red) and ER (green) shows the distribution of EpoR molecules outside and within the cytoplasmic membrane.

A2780
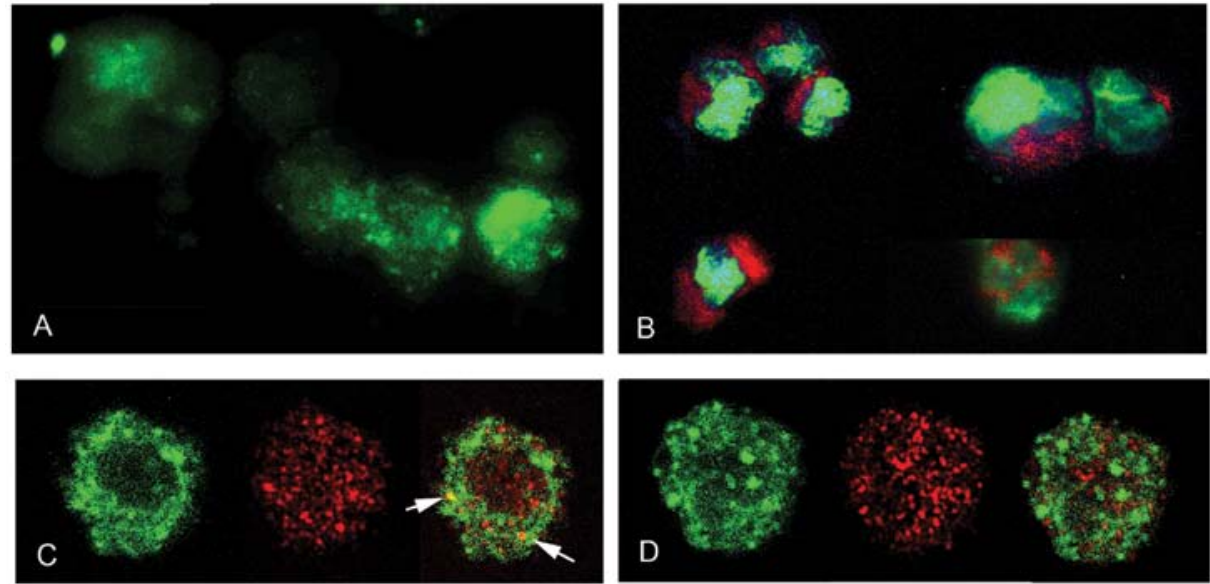

Figure 2. Immunocytochemical analysis of erythropoietin receptor and endoplasmic reticulum with secretory transport distribution in A2780 cells. (A) Single fluorescent staining using antibody to erythropoietin receptor (EpoR) and FITC-labelled secondary antibody. (B) Double staining of EpoR with biotinylated antihuman EpoR antibody (followed by incubation with streptavidin AlexaFluor 594 conjugate) and endoplasmic reticulum (ER) and secretory transport specific GFP labelled protein expressed following transduction of cells with CellLight reagents (see Materials and methods). Representative picture shows the presence of EpoR in the cytoplasm close to outside surface of ER. (C and D) Optical sections of the cells after double staining of EpoR (red) and ER (green) and merged sections using confocal microscopy. (C) Images of the middle part of the cell indicated by the presence of nucleus. Note a few yellow patches on merged sections (arrow) which show where two molecules coincide. (D) Images of the frontal part of cells above nucleus and close to the cytoplasmic membrane, where co-localisation of cells was not seen.

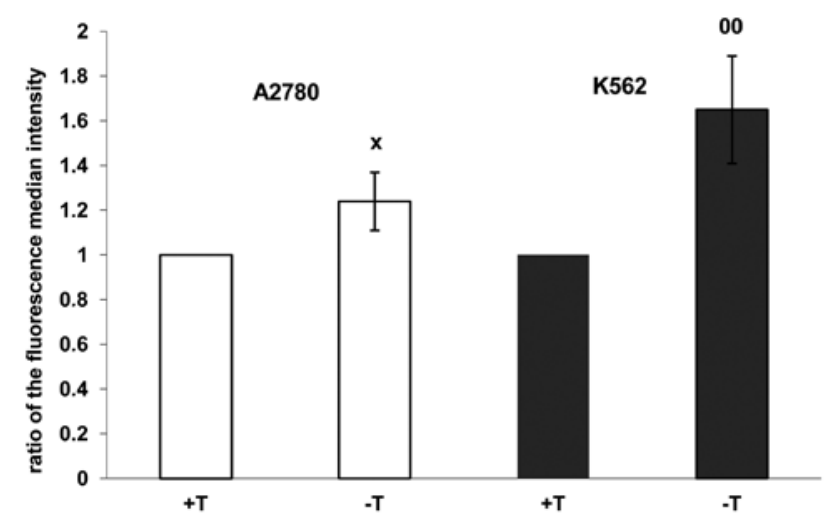

Figure 3. Presence of erythropoetin receptor on the surface of A2780 and K562 cells. The cells were incubated with fluorescein-conjugated anti-erythropoietin receptor (EpoR) antibody and analyzed by FACSCalibur flow cytometer. EpoR presence is expressed as the ratio of the fluorescence median intensity in nontrypsinized (-T) cells vs. trypsinized $(+\mathrm{T})$ control. Data are presented as means \pm $\mathrm{SD}$ of three independent experiments. The statistical significance is designated as follows: A2780 -T vs. A2780 + T ${ }^{\times} \mathrm{P}<0.05$; K562 -T vs. $\mathrm{K} 562+\mathrm{T}{ }^{\circ} \mathrm{P}<0.01$

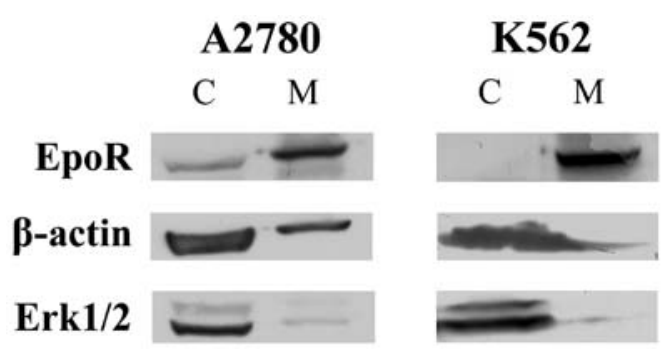

Figure 4. Presence of erythropoietin receptor in A2780 and K562 cytoplasm (C) and membrane $(\mathrm{M})$ protein fractions. The isolation of protein fractions was done by two independent techniques (see Materials and methods) and analyzed by Western blot analysis. Cross-contamination of protein fractions was double checked by detection of $\beta$-actin as well as Erk1/2 proteins. The isolation of protein fractions was repeated three times. Representative results.

EpoR had significantly lower proliferation monitored at 48, 72 and $96 \mathrm{~h}$ after seeding when compared to NTshRNA control 


$\begin{array}{rrrrrrr}\text { FCS }(0.1 \%) & - & + & + & + & + & + \\ \text { sEpoR } & - & - & - & - & + & + \\ \text { Epo }(I U / m l) & - & - & 5 & 50 & - & 50\end{array}$

P-Akt

Akt

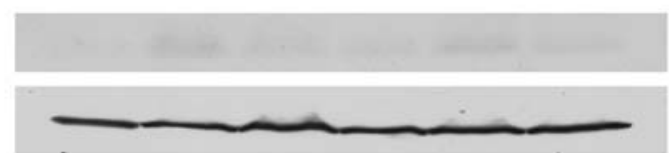

P-Erk1/2

Erk1/2

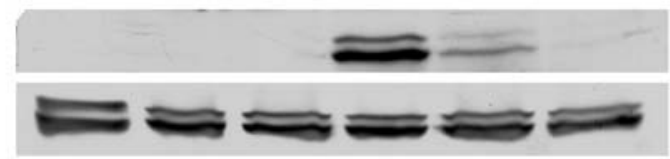

Figure 5. Erythropoietin induced signalization in A2780 cells. Western blot analysis of phosphorylated vs. normal Akt and Erk1/2 proteins in whole-A2780 cell lysates prepared after overnight serum starvation as well as treatment of A2780 cells with erythropoietin (Epo) and/or soluble erythropoietin receptor (sEpoR). Any phosphorylation signal was detected after vehicle $[0.1 \%$ fetal calf serum (FCS)] treatment of A2780 cells. The experiment was repeated three times. Representative results.

as well as normal A2780 cells (Fig. 7). This result suggests a paracrine and/or autocrine mechanism.

\section{Discussion}

Cytoplasm localization of EpoR has rarely been demonstrated. First Yoshimura et al (13) reported that $\mathrm{Ba} / \mathrm{F} 3$ cells transfected with the EpoR cDNA expressed very little EpoR on the cell surface. However, transfection with EpoR cDNA and with

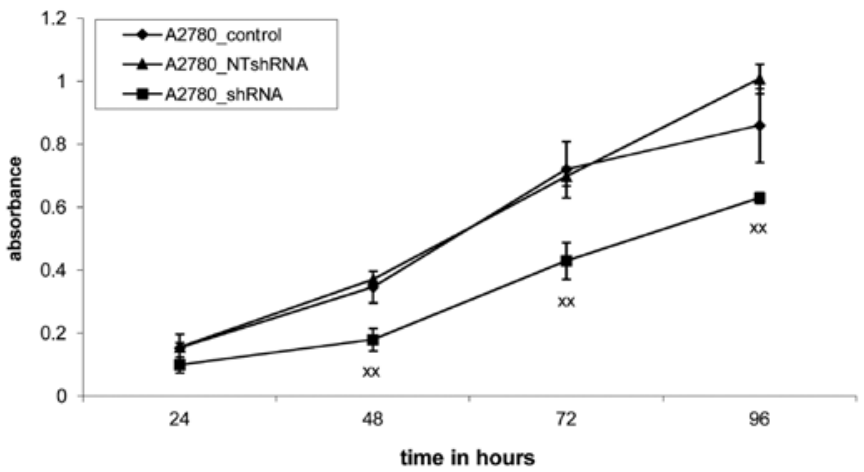

Figure 7. Silencing of erythropoietin receptor expression slowed down proliferation of A2780 cells. The proliferation of control cells, transduced cells by the lentiviral short hairpin RNA (shRNA) targeting specific mRNA sequence of erythropoietin receptor (EpoR) as well as cells transduced by non-targeting shRNA vector (NTshRNA) were evaluated by MTT assay. The results are presented as means $\pm \mathrm{SD}$ of three independent experiments. The statistical significance is designated as follows: A2780_shRNA vs. A2780_control and A2780_NTshRNA ${ }^{x \times} \mathrm{P}<0.01$.

cDNA for Friend spleen focus-forming virus envelope glycoprotein gp55 resulted in a more stable form of EpoR (remained in rough endoplasmic reticulum) allowing $\mathrm{Ba} / \mathrm{F} 3$ cells to grow in the absence of Epo (13). Paragh et al (10) mentioned cytoplasmic localization of EpoR in A2780 cells without providing further detail. These authors, however, were not able to identify the receptor on the surface of A2780 cells by flow cytometry. In contrast, Miller et al (7) demonstrated cytoplasmic membrane localization of EpoR in A2780 cells using the same technique and the same antibody. In our previous study (14), we also
NTshRNA

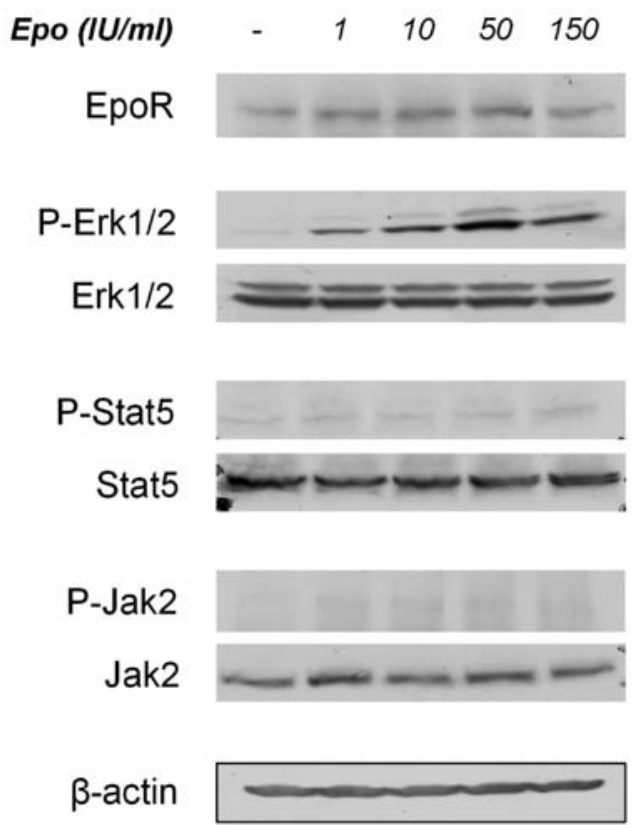

ShRNA
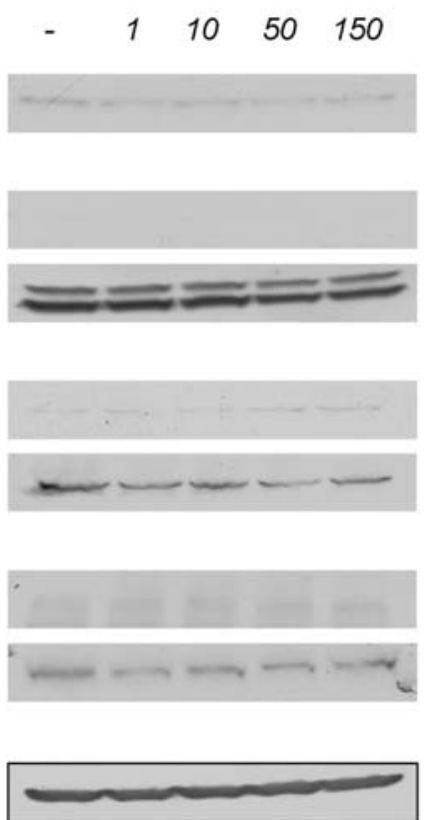

Figure 6. Loss of erythropoietin receptor signaling after its silencing with short hairpin RNA in A2780 cells. The cells transduced by the lentiviral short hairpin RNA (shRNA) targeting specific mRNA sequence of erythropoietin receptor (EpoR) as well as cells transduced by non-targeting shRNA vector (NTshRNA) were overnight serum starved and incubated with different concentrations of erythropoietin (Epo) for $10 \mathrm{~min}$. Total cell lysates were then analyzed by Western blot analysis for the presence of EpoR, phoshorylated-Erk1/2 (P-Erk1/2), Stat5 (P-Stat5), Jak2 (P-Jak2) and unphosphorylated Erk1/2, Stat5 and Jak2 proteins. Equal loading was confirmed by detection of $\beta$-actin. The experiment was repeated three times. Representative results. 
showed cell surface localization of EpoR in A2780. We have now found that there is even greater abundance of EpoR in the cytoplasm of A2780. Interestingly, only a small fraction of the EpoR was present in soluble form (cytoplasm fraction), whereas a far larger amount of EpoR was identified in the membrane fraction. It appears that in addition to the plasma membrane fraction, there are other membrane structures of A2780 cells located in cytoplasm where EpoR might potentially be internalized. Endoplasmic reticulum localization of EpoR in A2780 cells was suggested by our double staining and confocal microscopy.

The existence of multiple EpoR isoforms in human cancer cells that may modulate the cellular effects of recombinant human Epo has been discussed by Arcasoy et al (5). Our results confirm the existence of at least three EpoR forms in A2780 cells. The first is represented on the cytoplasm membrane, the second by the cytoplasmic form and the third is in cytoplasmic membrane structures.

We speculate that the cytoplasmic membrane EpoR may be functional, as evidenced by recombinant Epo induced Erk1/2 phosphorylation. The role of Erk1/2 in Epo induced signaling in A2780 cells was confirmed in our study by EpoR silencing, which resulted in down-regulation of Erk1/2 signaling and a reduction of A2780 proliferation. These results correlate well with the in vivo portion of the Paragh et al (10) study, where inhibition of EpoR expression led to abrogated A2780 tumor xenograft growth with decreased EpoR signaling. However, contrary to our findings is a study and its in vitro results, reporting that exogenous Epo did not stimulate EpoR-mediated signaling in A2780 cells (10). Both our study as well as that of Paragh et al (10) showed the identical effect of EpoR silencing on markedly reduced in vitro cell proliferation of A2780 cells. Contrary to both our and Paragh et al (10) studies are findings of Swift et al (8), of no effect of EpoR knockdown on the viability of A2780 or SKOV-3 cells. The discrepancy in EpoR functionality results could be explained by clonal differences in one cell line, by its different culturing (inactivated or regular serum) and/or experimental conditions $(8,15)$.

\section{Acknowledgements}

This study was supported by the Slovak Research and Development Agency under contract no. VVCE-0001-07 and LPP-0062-09 and the Scientific Grant Agency of the Ministry of Education of the Slovak Republic under contract nos. VEGA 1/0296/09, VEGA 1/0733/12 and the NEXO
(Network of Excellence in Oncology) under contract no. ITMS 26220120024.

\section{References}

1. Arcasoy MO: The non-haematopoietic biological effects of erythropoietin. Br J Haematol 141: 14-31, 2008.

2. Bennett CL, Lai SY, Henke M, Barnato SE, Armitage JO and Sartor O: Association between pharmaceutical support and basic science research on erythropoiesis-stimulating agents. Arch Intern Med 170: 1490-1498, 2010.

3. Bohlius J, Tonia T and Schwarzer G: Twist and shout: one decade of meta-analyses of erythropoiesis-stimulating agents in cancer patients. Acta Haematol 125: 55-67, 2011.

4. McKinney M and Arcasoy MO: Erythropoietin for oncology supportive care. Exp Cell Res 317: 1246-1254, 2011.

5. Arcasoy MO, Jiang X and Haroon ZA: Expression of erythropoietin receptor splice variants in human cancer. Biochem Biophys Res Commun 307: 999-1007, 2003.

6. Um M, Gross AW and Lodish HF: A 'classical' homodimeric erythropoietin receptor is essential for the antiapoptotic effects of erythropoietin on differentiated neuroblastoma SH-SY5Y and pheochromocytoma PC-12 cells. Cell Signal 19: 634-645, 2007.

7. Miller CP, Lowe KA, Valliant-Saunders K, et al: Evaluating erythropoietin-associated tumor progression using archival tissues from a phase III clinical trial. Stem Cells 27: 2353-2361, 2009.

8. Swift S, Ellison AR, Kassner P, et al: Absence of functional EpoR expression in human tumor cell lines. Blood 115: 4254-4263, 2010.

9. Jeong JY, Feldman L, Solar P, Szenajch J and Sytkowski AJ: Characterization of erythropoietin receptor and erythropoietin expression and function in human ovarian cancer cells. Int $\mathrm{J}$ Cancer 122: 274-280, 2008.

10. Paragh G, Kumar SM, Rakosy Z, Choi SC, Xu X and Acs G: RNA interference-mediated inhibition of erythropoietin receptor expression suppresses tumor growth and invasiveness in A2780 human ovarian carcinoma cells. Am J Pathol 174: 1504-1514, 2009.

11. Johnstone A and Thorpe R: In: Immunochemistry in Practice. Blackwell Science Ltd., Berlin, 1996.

12. Griffiths LG, Choe L, Lee KH, Reardon KF and Orton EC: Protein extraction and 2-DE of water- and lipid-soluble proteins from bovine pericardium, a low-cellularity tissue. Electrophoresis 29: 4508-4515, 2008

13. Yoshimura A, D'Andrea AD and Lodish HF: Friend spleen focusforming virus glycoprotein gp55 interacts with the erythropoietin receptor in the endoplasmic reticulum and affects receptor metabolism. Proc Natl Acad Sci USA 87: 4139-4143, 1990.

14. Solar P, Feldman L, Jeong JY, Busingye JR and Sytkowski AJ: Erythropoietin treatment of human ovarian cancer cells results in enhanced signaling and a paclitaxel-resistant phenotype. Int J Cancer 122: 281-288, 2008.

15. Fu P, Jiang X and Arcasoy MO: Constitutively active erythropoietin receptor expression in breast cancer cells promotes cellular proliferation and migration through a MAP-kinase dependent pathway. Biochem Biophys Res Commun 379: 696-701, 2009. 